\title{
ANTENNA SELECTION FOR OPPORTUNISTIC INTERFERENCE MANAGEMENT IN MIMO BROADCAST CHANNELS
}

\author{
Lemonia Dritsoula $^{\dagger}$, Zheng Wang ${ }^{\dagger}$, Hamid R. Sadjadpour ${ }^{\dagger}$, and J.J. Garcia-Luna-Aceves ${ }^{\ddagger}$ \\ ${ }^{\dagger}$ Department of Electrical Engineering and ${ }^{\ddagger}$ Computer Engineering \\ University of California, Santa Cruz, 1156 High Street, Santa Cruz, CA 95064, USA \\ ‡ Palo Alto Research Center (PARC), 3333 Coyote Hill Road, Palo Alto, CA 94304, USA \\ Email: $\{$ lenia, wzgold, hamid, jj\}@ soe.ucsc.edu
}

\begin{abstract}
Opportunistic interference management (OIM) is an approach that can asymptotically achieve dirty paper coding (DPC) capacity in the downlink of wireless cellular networks with minimum feedback requirement. With $K$ antennas at the base station and $M$ mobile users in the cell, the proposed technique requires only $K$ integer numbers related to channel state information (CSI). This multiplexing gain of $K$ is achieved at the expense of $M$ mobile users such that $K=\Theta(\log M)$. We introduce an antenna selection scheme at the base station to reduce the minimum number of required mobile users significantly at the expense of reasonable increase in feedback.
\end{abstract}

\section{INTRODUCTION}

Multiuser diversity scheme [1] is an alternative approach to more traditional techniques like time division multiple access (TDMA) to increase the capacity of wireless cellular networks. The main idea behind this approach is that the base station selects a mobile user with the best channel condition by taking advantage of the time varying nature of fading channels, thus maximizing the signal-to-noise ratio (SNR). Traditionally, fading and interference have been viewed as the two major impeding factors in increasing the capacity of wireless cellular networks. The Opportunistic interference management (OIM) scheme is an approach that takes advantage of the fading in the channel to reduce the negative effects of interference.

Several schemes have been developed for MIMO broadcast channels that achieve optimal dirty paper coding capacity by using random beamforming [2,3]. Most recent studies [4-7] have investigated the effect of partial finite-rate feedback on the capacity of MIMO broadcast channels in networks with limited number of users $M$.

We present the OIM [8] technique for the downlink of wireless cellular networks, in which $d^{1}(d \leq K)$ independent data streams can be broadcasted to $d(d \ll M)$ mobile stations with single antenna such that these data streams do not

\footnotetext{
${ }^{1} d$ is a random variable.
}

interfere significantly with each other. Furthermore, the mean value of $d$, i.e. $D=\mathbf{E}[d]$, can be any number up to the maximum value of $K$ as long as $M$ is large enough [8]. Therefore, OIM is capable of achieving the maximum multiplexing gain as long as there are enough mobile stations in the network. The feedback requirement to transmit $K$ independent data streams is proportional to $K$ [8]. The original multiuser diversity concept was based on searching for the best channel to communicate, while our approach shows that searching simultaneously for the best and worst channels can lead to significant capacity gains. This technique can asymptotically achieve the capacity of DPC when $M$ is very large.

The OIM scheme does not require mobile stations to cooperate for synchronization during transmission. It achieves optimal $K$ maximum multiplexing gain in the downlink of cellular systems as long as $K=\Theta(\log M)$. However, in most practical cellular networks, there may not be so many mobiles users in a cell. Therefore, it is important to reduce the minimum required number of mobile users. This paper introduces an antenna selection technique at the base station such that it reduces the minimum required number of mobile users significantly. This improvement is achieved at the expense of modest increase in the feedback requirement and additional computational complexity at the base station receiver.

The remainder of the paper is organized as follows. In Section 2 we present an overview of the previous work. The system model and problem formulation are presented in Section 3. Section 4 focuses on our new antenna selection scheme and the lower and upper bounds computation of multiplexing gain as a function of $M$. The simulation results are shown in Section 5. Finally, we conclude the paper and discuss about future work in Section 6.

\section{PREVIOUS WORK}

DPC provides the optimal $K \log \log M$ sum-rate capacity which is the maximum multiplexing and multiuser diversity gains. These gains are achieved at the expense of full CSI requirement and infinite-rate feedback $M$ when $M$ tends to infin- 
ity. Sharif and Hassibi's work [2,3] is based on the random beamforming concept to search for the best SINR in the network. The feedback requirement in their approach is $M$ complex numbers instead of full CSI information. This scheme achieves the same capacity of $K \log \log M$ similar to DPC when $M$ goes to infinity.

The OIM technique [8] provides a new scheduling scheme which requires only minimum finite-rate feedback $K$ and yet retains the optimal multiplexing and multiuser diversity gains achievable by dirty paper coding. To the best of our knowledge, [9] and [4] are the only two publications with some similarities to our approach. Diaz et al. [9] proposed "1-bit" feedback from the mobile users instead of CSI information to the base station with the total feedback still proportional to $M$. While Tajer et al.'s [4] scheduling scheme is asymptotically optimal, it also exhibits a good performance for practical network sizes. They also showed [4] that by appropriate design of the feedback mechanism, they can refrain the aggregate feedback from increasing with the number of mobile users and for asymptotically large networks, the total number of feedback is bounded by $K \log K$ bits.

\section{NETWORK MODEL AND PROBLEM FORMULATION}

\subsection{Network model}

We consider a network setting that involves a base station with $K$ antennas and $M$ mobile users. We investigate the problem of optimal transmission in the downlink of a cellular network when the base station can transmit up to $K$ concurrent signals. Our intention is to achieve these gains with minimum required number of mobile users $M$ using a new antenna selection scheme. We assume that all mobile users are equipped with a single antenna.

The channel between the base station and mobile stations $\mathbf{H}$ is an $M \times K$ matrix. We consider block fading model where the channel coefficients are constant during coherence interval of $T$. The noise at each of the receive antennas is i.i.d. with $\mathcal{C N}\left(0, \sigma_{n}^{2}\right)$ distribution. The above described network model is depicted in Fig. 1, where the dotted and solid lines represent the weak and strong channels equivalently.

\subsection{Description of the problem}

We first review the method developed in [8] and discuss its main drawback: the logarithmic relationship between $K$ and $M$. The main contribution of this paper is to reduce the minimum required number of mobile users $M$ while achieving the maximum multiplexing gain.

The communication in OIM technique takes place in two phases. During the first phase, the base station antennas sequentially transmit $K$ pilot signals. In this period, all the mobile stations listen to these known messages. After the last pilot signal is transmitted, mobile stations evaluate the SNR

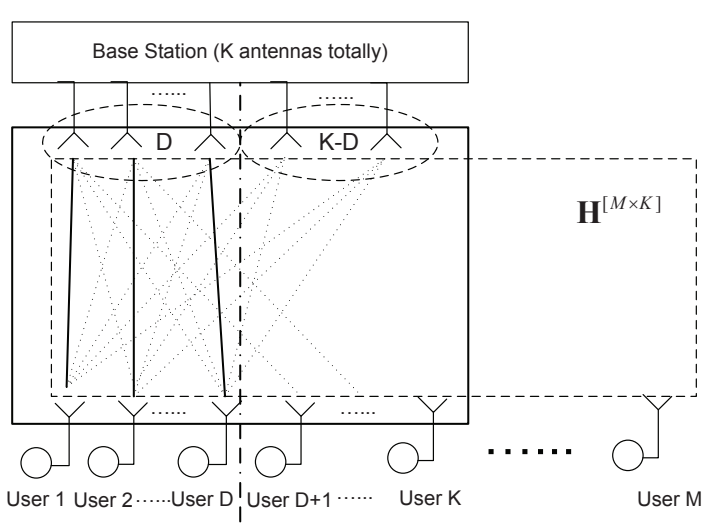

Fig. 1. Wireless cellular network model

for each antenna. If the SNR for only one transmit antenna is greater than a pre-determined threshold $\mathrm{SNR}_{t r}$ and below another pre-determined threshold of $\mathrm{INR}_{t r}$ for the remaining $K-1$ antennas, that particular mobile station will select that particular antenna at the base station.

Given that more than one mobile station may be found with this property, in the second phase of communication, the mobile stations notify the base station that they have the required criterion to receive packets during the remaining time period of $T$. We assume there is a channel access protocol for these mobile stations to contact the base station and also the base station will resolve the case when two mobile stations have similar property for the same antenna by some protocol.

Note that, if we choose appropriate values for $\mathrm{SNR}_{t r}$ and $\mathrm{INR}_{t r}$ such that $\mathrm{SNR}_{t r} \gg \mathrm{INR}_{t r}$, then the base station can simultaneously transmit different packets from its antennas to different mobile stations. The mobile stations only receive their respective packets with a strong signal and can treat the rest of the signals as noise. The value of $\mathrm{SNR}_{t r}\left(\right.$ or $\left.\mathrm{INR}_{t r}\right)$ can be selected as high (or low) as required for a given system, as long as $M$ is large enough.

Suppose that there are on average $D$ antennas that can be matched to corresponding mobile stations with the above property. Further, we select another $K-D$ mobile stations such that they do not have the above property and require cooperation among themselves to decode the $K-D$ data streams. Note that these $K-D$ nodes can potentially operate similar to a distributed MIMO system.

It has been proved in [8] that when $K=\Theta(\log M)$, then OIM achieves DPC asymptotic capacity. However, from practical point of view, usually there exists far less number of mobile users in a base station. Similar relationship was also reported for random beamforming technique in [3]. From practical point of view, it is important to achieve average multiplexing gain of $D$ for some small values of $M$. This paper introduces an antenna selection technique that achieves this goal at the expense of modest increase in the number of feedback requirement and some additional complexity at the base 
station.

\section{ANTENNA SELECTION ALGORITHM}

Antenna selection diversity [10] is a low-cost low-complexity alternative to capture many of the advantages of MIMO systems by choosing the path with the highest SNR among all channels between the base station antennas and a mobile user. In our approach, we assume that an average multiplexing gain of $D$ is desired while there are actually $K$ antennas at the base station such that $K \gg D$. Further, we define a new parameter $L$ such that it is the minimum number of channels between base station antennas and a single mobile user that their SNR is below $\mathrm{INR}_{t r}$ (weak channels).

Unlike the original OIM technique, we no longer require the mobile users to send their information when they have one strong channel and $K-1$ weak channels. Under the new scheme, each mobile user that has at least one strong channel and at least $L$ weak channels, sends its information to the base station. Under the new scheme, the mobile users should notify which channels are strong, which ones are weak and perhaps some channels are neither strong nor weak channel. Hence, each mobile user responds with more additional information than the original OIM technique.

There are mainly two ways to carry out this search. The optimum search is based on the exhaustive search among all possible combinations of mobile users such that we obtain the maximum multiplexing gain. This exhaustive search can be carried in practice using backtracking algorithms [11]. Backtracking is a general algorithm for finding all (or some) solutions to some computational problem, that incrementally builds candidates to the solutions, and abandons each partial candidate as soon as it determines that it cannot possibly lead to a valid or the best solution. This algorithm actually searches among all the different combinations of channels and selects the ones that results in maximum parallel transmissions. The disadvantage of the optimal solution is significant computational complexity at the base station and the time required to complete the search.

Our proposed sub-optimal approach is inspired based on antenna selection techniques. Having large number of antennas at the base station is a reasonable assumption and we need to select a subset of these antennas such that an equal number of mobile users have OIM capability with respect to these antennas. Each mobile user that has the OIM constraint with at least $L$ antennas at the base station, then that particular mobile user sends its information to the base station. Among all the mobile users that send their feedback information, we select the user with the largest number of antennas satisfying the OIM constraint. We create a table with the number of antennas related to this mobile user as values of the first row $^{2}$.

\footnotetext{
${ }^{2}$ If there are more than one mobile user with the largest number of antennas satisfying the OIM constraint, then we choose all of them and apply the parallel search for each one to find the best solution.
}

Then based on this set, we select the next mobile user that has the largest subset of this set. Note that the second mobile user satisfies the OIM constraint for this subset. We continue this algorithm until we find a group of mobile users that satisfies this scheme and have the largest multiplexing gain. In the original OIM approach, we have proved analytically [8] that the number of mobile users sending feedback to the base station is less than $K$ with probability going to 1 . The proposed antenna selection scheme will increase the feedback and there is clearly a tradeoff between minimum required mobile users for a given multiplexing gain and feedback requirement.

\subsection{Theoretical Analysis}

In this section, we derive an expression for the upper and lower bounds of the minimum required mobile users as a function of multiplexing gain based on the optimal search.

Let's define $\mathrm{SNR}_{j i}$ as the signal-to-noise ratio when antenna $j$ at the base station is transmitting packet to mobile station $i$ in the downlink. Further denote $\operatorname{INR}_{j i}$ as the interferenceto-noise ratio between transmit antenna $j$ at the base station and receiver mobile station $i$. The objective of OIM scheme is to find $D$ mobile stations out of $M$ choices that satisfy the following criterion.

\section{Strong Channel Case:}

$$
\begin{aligned}
& \mathrm{SNR}_{i i} \geq \mathrm{SNR}_{\mathrm{tr}}, 1 \leq i \leq D, \\
& \mathrm{INR}_{j i} \leq \mathrm{INR}_{\mathrm{tr}}, 1 \leq i, j \leq D, j \neq i
\end{aligned}
$$

The probability that a mobile user satisfies condition in (1) is given by

$$
\begin{aligned}
\operatorname{Pr}(A) & =\left(\begin{array}{c}
D \\
1
\end{array}\right) \int_{\mathrm{SNR}_{\mathrm{tr}}}^{\infty} p(z) d z\left(\int_{0}^{\mathrm{INR}_{\mathrm{tr}}} p(z) d z\right)^{D-1} \\
& =\left(\begin{array}{c}
D \\
1
\end{array}\right) e^{-\frac{\mathrm{SNR}_{\mathrm{tr}}}{\sigma}}\left(1-e^{-\frac{\mathrm{INR}_{\mathrm{tr}}}{\sigma}}\right)^{D-1}
\end{aligned}
$$

It can be proved [8] that the probability that $x$ mobile users satisfy OIM condition is a binomial distribution.

$$
\operatorname{Pr}(X=x)=\left(\begin{array}{c}
M \\
x
\end{array}\right)(\operatorname{Pr}(A))^{x}(1-\operatorname{Pr}(A))^{M-x}
$$

The average value of this binomial distribution is $M \cdot \operatorname{Pr}(A)$. Note that there are $\left(\begin{array}{l}K \\ D\end{array}\right)$ possible choices to satisfy $D$ antennas with constraints in Eq. (1). Therefore for the first user, there are $\left(\begin{array}{l}K \\ D\end{array}\right) M \operatorname{Pr}(A)$ possible users that may satisfy OIM condition. Once the first user is selected, the remaining users must satisfy the OIM condition only for these $D$ antennas. Hence, there are on average $\frac{D-1}{D} \cdot M \cdot \operatorname{Pr}(A)$ possible users that can be selected as the second user, $\frac{D-2}{D} \cdot M \cdot \operatorname{Pr}(A)$ possible users that can be selected as the third user and finally $\frac{1}{D} \cdot M \cdot \operatorname{Pr}(A)$ possible users that can be selected as the last user. Note that the number of possible users that can be selected as the first 


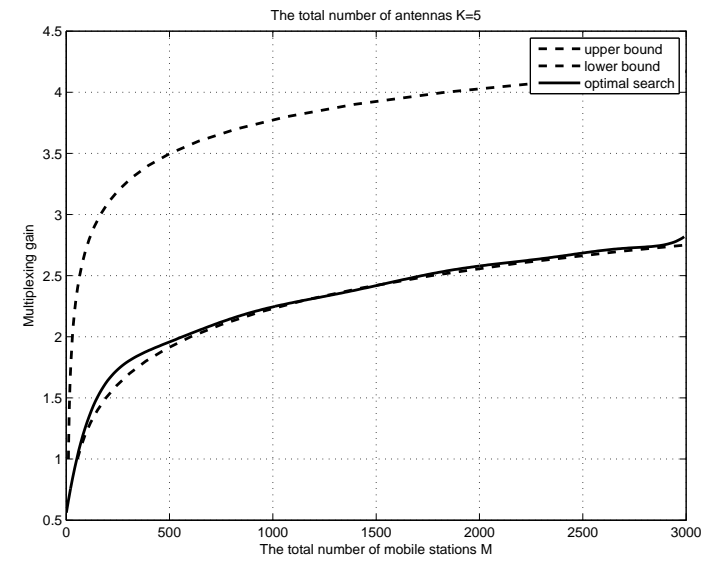

Fig. 2. Upper and lower bounds vs. optimal search algorithm for $\mathrm{K}=5$

(or last) user can provide an upper (or lower) bound on the average number of users satisfying the OIM condition.

Based on these observations, the lower bound for the expected value of $D$ is given by

$$
D=\mathbf{E}(d) \geq \frac{M}{D} \cdot \operatorname{Pr}(A)
$$

Thus,

$$
M \leq D^{2} \cdot(\operatorname{Pr}(A))^{-1} .
$$

The upper bound is given by

$$
D=\mathbf{E}(d) \leq M \cdot\left(\begin{array}{l}
K \\
D
\end{array}\right) \cdot \operatorname{Pr}(A) .
$$

Equivalently, we have

$$
M \geq D \cdot(\operatorname{Pr}(A))^{-1} \cdot\left(\left(\begin{array}{l}
K \\
D
\end{array}\right)\right)^{-1} .
$$

Finally, we can combine the above results into the following equation:

$$
D \cdot(\operatorname{Pr}(A))^{-1} \cdot\left(\left(\begin{array}{l}
K \\
D
\end{array}\right)\right)^{-1} \leq M \leq D^{2} \cdot(\operatorname{Pr}(A))^{-1}
$$

\section{NUMERICAL RESULTS}

Fig. 2 compares our analytical lower and upper bounds of $M$ with simulation results as a function of $D$ when $\mathrm{SNR}_{t r}=40$, $K=5, \mathrm{INR}_{t r}=2$ and $\sigma=10$. The solid line which represents the simulation results is based on optimal exhaustive search to achieve maximum multiplexing gain with minimum number of mobile users. It is clear from simulation results that the lower bound is a tight bound while the upper bound is a loose bound.

Figures 3 and 4 compare the performance of our suboptimal search for different values of $L$ with the optimal search

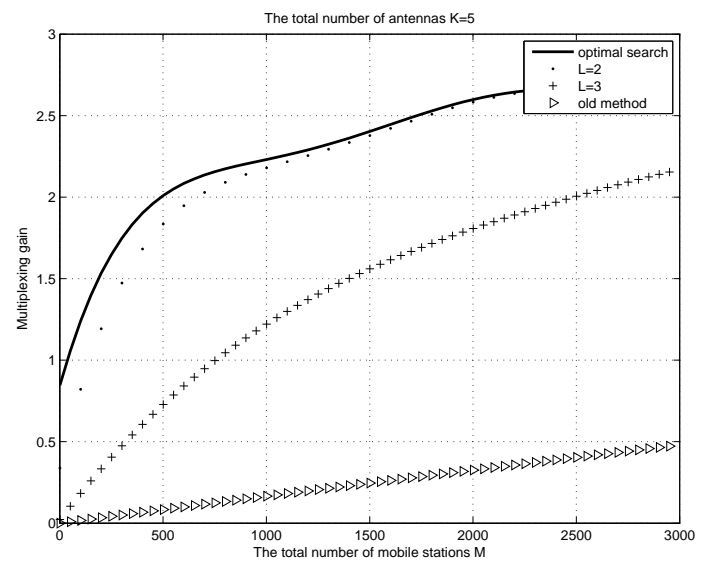

Fig. 3. Trade off between multiplexing gain and minimum number of users required for $\mathrm{K}=5$

and our original OIM scheme [8] for $K=5$ and $K=10$ respectively. It is clear from these results that our proposed antenna selection technique reduces the minimum required number of mobile users significantly. In addition, the feedback is slightly increased and its value depends on how strict or loose the OIM conditions are. For example for $D=1$ and $K=5$, the optimal search requires only 30 mobile users and feedback of 3 integers, while the suboptimal search requires 42 or 124 mobile users and feedback of 2 , when $L=2$ or $L=3$ respectively. When $K=10, D=1$ and for the same set of parameters as before, the optimal search requires 15 users while the suboptimal search requires 22,32 , and 56 users and feedback of 3,4 and 3 users for $L=1, L=2$, and $L=3$ respectively.

One intriguing question is why a multiplexing of one, i.e. $D=1$, is important, since we would just need a single mobile user to achieve this gain. The answer relies on the following facts. First, in the OIM scheme a multiplexing gain of one means that there is at least one antenna at the base station that has a deep fade with that particular user. Since the selection of mobile users that have OIM capability is completely random and depends on the time-varying fading nature of the channel, then the problem of fairness becomes a major issue.

However, this technique can be incorporated into the current wireless standards. For example, in a TDMA system we can use $L$ antennas at the base station for regular TDMA communication since their signal is extremely weak at the receiver of mobile users that are participating in OIM, i.e., they are not interfering with those transmissions. On the other hand, nodes utilizing OIM can affect TDMA receiver but we have shown recently [12] that they can be orthogonalized at the TDMA receiver side using a technique that does not require any channel knowledge. This technique is fundamentally different from beamforming concept which has been described in details in [12]. 


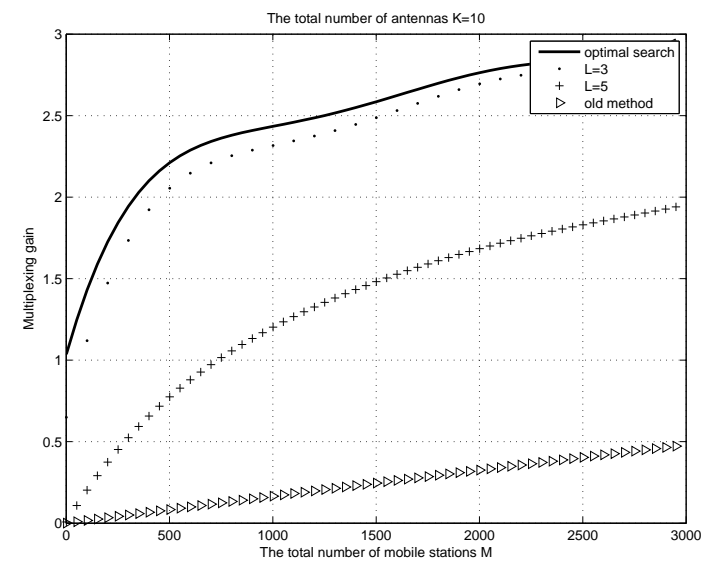

Fig. 4. Trade off between multiplexing gain and minimum number of users required for $\mathrm{K}=10$

\section{CONCLUSIONS}

In this paper, we investigated the effect of using antenna selection techniques for an OIM scheme to increase the multiplexing gain in a multi-user environment. Analytical upper and lower bounds for the minimum number of mobile users as a function of multiplexing gain are derived. Both our analytical and numerical results show that by using this approach, higher multiplexing gains with fewer users can be achieved. It has been shown through simulation that with as few as 19 mobile users, we can implement OIM technique in the downlink of wireless cellular systems.

\section{Acknowledgment}

This research was partially sponsored by the U.S. Army Research Laboratory under the Network Science Collaborative Technology Alliance, Agreement Number W911NF-09-0053, by the Army Research Office under agreement number W911NF05-1-0246, by the National Science Foundation under grant CCF-0729230, and by the Baskin Chair of Computer Engineering. The views and conclusions contained in this document are those of the author(s) and should not be interpreted as representing the official policies, either expressed or implied, of the U.S. Army Research Laboratory or the U.S. Government. The U.S. Government is authorized to reproduce and distribute reprints for Government purposes notwithstanding any copyright notation hereon.

\section{REFERENCES}

[1] R. Knopp and P. Humblet, "Information capacity and power control in single-cell multiuser communications," in Proc. of IEEE ICC 1995, Seattle, Washington, USA, June 18-22 1995.
[2] M. Sharif and B. Hassibi, "A comparison of timesharing, DPC, and beamforming for MIMO broadcast channels with many users," IEEE Transactions on Communications, vol. 55, no. 1, pp. 11-15, January 2007.

[3] _ _ " "On the capacity of MIMO broadcast channels with partial side information," IEEE Transactions on Information Theory, vol. 51, no. 2, pp. 506-522, February 2005.

[4] A. Tajer and X. Wang, "Opportunistic multi-antenna downlink transmission with finite-rate feedback," in Proc. of Allerton 2008, Monticello, Illinois, USA, September 23-26 2008 .

[5] T. Yoo, N. Jindal, and A. Goldsmith, "Multi-antenna downlink channels with limited feedback and user selection," IEEE Journal on Selected Areas in Communications, vol. 27, no. 7, p. 14781491, September 2007.

[6] K. Huang, R. W. Heath, and J. G. Andrews, "Space division multiple access with a sum feedback rate constraint," IEEE Transactions on Signal Processing, vol. 55, no. 7, p. 38793891, July 2007.

[7] S. Sanayei and A. Nosratinia, "Opportunistic beamforming with limited feedback," IEEE Transactions on Wireless Commununications, vol. 6, no. 8, p. 2765 2771, August 2007.

[8] Z. Wang, M. Ji, H. Sadjadpour, and J. J. Garcia-LunaAceves, "Cooperation-multiuser diversity tradeoff in wireless cellular networks," in Proc. of IEEE GLOBECOM 2009, Honolulu, Hawaii, USA, November 30 December 42009.

[9] J. Diaz, O. Simeone, and Y. Bar-Ness, "Asymptotic analysis of reduced-feedback strategies for MIMO Gaussian broadcast channels," IEEE Transactions on Information Theory, vol. 54, no. 3, pp. 1308 - 1316, March 2008.

[10] S. Sanayei and A. Nosratinia, "Antenna selection in mimo systems," IEEE Comm. Mag., vol. 42, no. 10, pp. 68-73, October 2004.

[11] G. Brassard and P. Bratley, Fundamentals of Algorithmics. Prentice-Hall, 1995.

[12] Z. Wang, M. Ji, L. Dritsoula, H. Sadjadpour, and J. J. Garcia-Luna-Aceves, "On the minimum side information of mimo broadcast channel," in Proc. of European Wireless Conference, Lucca, Italy, April 12 - April 15 2010. 